\title{
The impact of immigration detention on mental health: a systematic review
}

\author{
M. von Werthern ${ }^{1}$, K. Robjant ${ }^{1}$, Z. Chui ${ }^{1}$, R. Schon ${ }^{1}$, L. Ottisova ${ }^{2}$, C. Mason ${ }^{1}$ and C. Katona ${ }^{1,3^{*}}$ (D)
}

\begin{abstract}
Background: The number of asylum seekers, refugees and internally displaced people worldwide has increased dramatically over the past 5 years. Many countries are continuing to resort to detaining asylum seekers and other migrants, despite concerns that this may be harmful. In light of the considerable body of recent research, this review aims to update and expand on a 2009 systematic review on the mental health consequences of detention on adult, adolescent and child immigration detainees, which found (on the basis on 9 studies) that there was consistent evidence that immigration detention had adverse effects on mental health.

Methods: Three databases were searched using key terms relating to immigration detention and mental health. Electronic searches were supplemented by reference screening. Studies were included if they were quantitative, included individuals detained for immigration purposes, reported on mental health problems and were published in peer-reviewed journals. Two reviewers independently screened papers for eligibility, and a further two reviewers completed quality appraisals for included studies.
\end{abstract}

Results: Twenty- six studies (21 of which were not included in the 2009 review) reporting on a total of 2099 participants were included in the review. Overall, these studies indicated that adults, adolescents and children experienced high levels of mental health problems. Anxiety, depression and post-traumatic stress disorder were most commonly reported both during and following detention. Higher symptom scores were found in detained compared to non-detained refugees. In addition (and more clearly than was evident in 2009), detention duration was positively associated with severity of mental symptoms. Greater trauma exposure prior to detention was also associated with symptom severity.

Conclusions: The literature base reviewed in this paper consistently demonstrated severe mental health consequences amongst detainees across a wide range of settings and jurisdictions. There is a pressing need for the proper consideration of mental health and consequent risk of detention-related harm in decisions surrounding detention as well as for improved care for individuals within detention facilities. Recommendations based on these findings are presented, including increased focus on the identification of vulnerability and on minimising the duration of detention.

Keywords: Mental health, Vulnerability, Immigration detention, Asylum seekers, Refugees

\section{Background}

The number of people forcibly displaced worldwide as a result of persecution, conflict, generalized violence or human rights violations has increased dramatically in the last 5 years. The UNHCR estimates that there are currently (July 2018) 68.5 million forced migrants around the world [1].

\footnotetext{
* Correspondence: cornelius@helenbamber.org

${ }^{1}$ Helen Bamber Foundation, Bruges Place, 15-20 Baynes Street, London NW1 OTF, UK

${ }^{3}$ Division of Psychiatry, University College London, London, UK

Full list of author information is available at the end of the article
}

Many countries detain asylum seekers in immigration detention centres whilst their applications are processed or following a decision to refuse them protection. At present the total number of third-country nationals held in immigration detention in the European Union is estimated to be 158,792 [2]. In the UK alone, this figure is 32526 people, representing 20\% of total EU detainees, despite a much smaller percentage of asylum applications.

There is robust and consistent evidence that refugees are more vulnerable to mental illness, particularly depression and PTSD, as compared to the general

(c) The Author(s). 2018 Open Access This article is distributed under the terms of the Creative Commons Attribution 4.0 International License (http://creativecommons.org/licenses/by/4.0/), which permits unrestricted use, distribution, and 
population [3, 4]. Recent evidence indicates that vulnerability to non-affective psychoses is also increased in refugees compared to other migrants, reflecting their severe and often repeated exposure to adversity [5]. In addition to pre-migration factors such as exposure to torture or human trafficking, post-migration factors, including prolonged asylum procedures, prohibition from working, poverty and poor housing are significantly associated with poor mental health [6-9]. Research from the UK also indicates that post-migration and asylum-related stressors are associated with PTSD symptoms and emotional distress in asylum seekers and refugees [10].

Time spent in immigration detention in the host country is a particular post-migration stressor that entails loss of liberty and the threat of forced return to the country of origin. For many asylum seekers with a history of major trauma, it is reminiscent of contexts in their country of origin where they had been deprived of their liberty and human rights [11]. Immigration detention also exposes asylum seekers to possible abuse from staff and violence from fellow detainees, social isolation, and forceful removal. In the UK, the Medical Justice charity has documented these problems in a series of reports [12].

The practice of detaining asylum seekers, a group with a pre-existing vulnerability to mental health problems due to higher exposure to trauma pre- and peri-migration, [4] risks further exacerbating their mental health difficulties. The experience of detention may act as a new stressor, which adds to the cumulative effect of exposure to trauma, leading to an increased likelihood of developing mental health difficulties such as PTSD as a result of the 'building block effect' [13]. Indeed, a 2009 systematic review reporting on the effects of immigration detention on mental health found detainees to have high levels of anxiety, depression and post-traumatic stress disorder [14]. Suicidal ideation and deliberate self-harm were also common. Severity of distress was significantly greater in those who had been detained for relatively long periods [14]. The studies reviewed outlined the extent and severity of the mental health problems observed in detained people. The authors noted this research area was still in its infancy and highlighted the urgent need for further research on both the acute and longer-term impact of immigration detention on mental health, as well as its independence or otherwise from other risk factors.

Since 2009, a number of new studies have been published in the context of rising levels of forced migration globally and the frequent use of immigration detention to manage the increasing numbers of asylum seekers.

These findings have led to increasing concern that immigration detention may constitute a significant component of the post-migration adversity experienced by some asylum seekers. Such concerns over the treatment, support and management of both children and adults within detention facilities have been continuously expressed by numerous institutions across several countries, with many calling for legislative amendments to end immigration detention, particularly of children [1517]. More recently, the UNHCR has also voiced concern over the physical and psychological effects of possible mandatory detention of all asylum seekers in Hungary [18]. The European Commission have reviewed the (very variable) use of alternatives to detention across the European Community. [19]

There has also been very extensive recent concern at the potential adverse consequences and human rights implications of the separation of migrant children from their parents by the USA authorities [20]. This led to this practice being discontinued [21].

In 2002, Australia was unique in its policy of indefinite, non-reviewable, mandatory detention of asylum seekers without valid entry documents arriving by boat. Clinical observation raised initial concerns, followed by the publication of research demonstrating the high prevalence of mental disorder in detention [22, 23]. Whereas the Australian authorities have since made changes to the law [24] that could be considered progress, the British government have sought to expand the capacity of immigration detention centres [25].

Although concerns have been raised over the mental health implications of immigration detention globally [26], we have taken the UK as a more in-depth example due to our familiarity with it and to the relatively large number of people detained there, incongruent with the comparatively small total number of asylum applications.

In 2015 a cross-party inquiry found that the UK detains too many people and for too long, and is more costly and less efficient than alternative systems operating in countries such as Australia and Sweden that have introduced community-based programmes to monitor asylum seekers and irregular migrants [27]. UK policy developments, therefore, provide an indicative illustration of these developing concerns.

In 2013, the Royal College of Psychiatrists issued a position statement on the detention of people with mental disorders in Immigration Removal Centres, which concluded that 'detention centres are likely to precipitate a significant deterioration of mental health in the majority of cases, greatly increasing both the suffering of the individual and the risk of suicide and self-harm' and therefore that 'people with mental disorder should only be subjected to immigration detention in very exceptional circumstances' [28].

In 2016, a government-commissioned review on the welfare of immigration detainees [29] highlighted the 'evident ethical, policy and practical implications' of 
medical research showing that immigration detention itself can seriously damage the mental health of detainees. A narrative review included within the report highlighted the particular vulnerability of people with a history of trauma and those with pre-existing mental or physical health problems to the adverse mental health effects of detention [30].

The UK Home Office published a Policy Guidance document on adults at risk in immigration detention [31]. which identified several 'conditions or experiences which will indicate that a person may be particularly vulnerable to harm in detention'. 'Immigration factors' enabled decisions to detain to be made and maintained despite substantial vulnerability factors being present. The Royal College of Psychiatrists issued a Position Statement expressing concern that the definition of torture within the new Adults at Risk policy added 'unnecessary and inappropriate complexity that does not assist in identifying those who are particularly vulnerable to the adverse effects of detention' [32]. In response to legal challenge, The UK Home Office has since changed this definition [33].

This review was conducted to provide a fuller and more up-to-date synthesis of the evidence. Its specific aims were to document (a) the prevalence and types of mental health problems among child and adult immigration detainees and (b) risk factors associated with mental health problems among immigration detainees. We looked particularly for evidence about the possible effects of pre-existing trauma and/or mental health problems and of duration of detention. Since detention systems vary between countries and nomenclature surrounding detainees vary across studies, the review takes a broad and inclusive stance and includes material on people detained within an immigration context (spanning asylum seekers, refugees and other migrants).

\section{Methods}

A systematic literature review following a modified version of the PRISMA guidelines [34] was conducted between October 2015 and May 2018 (PROSPERO Registration No: CRD42017056444).

\section{Inclusion and exclusion criteria}

Studies were included if they (a) included male or female adults or children detained for immigration purposes; (b) reported the prevalence of mental health problems; and (c) presented results of published peer-reviewed research based on one or more of the following study designs: cross-sectional survey; case-control study; cohort study; case series analysis; experimental study with baseline measures for the outcomes of interest; or secondary analysis of organisational records (Table 1). No restrictions were placed on country setting, country of origin of the immigration detainees, or method of measuring mental health outcomes. Since research in this area remains scarce, studies were not excluded on the grounds of their quality (though this is reported in Table 2). Qualitative studies will be presented in a subsequent paper.

Studies that did not report specifically on people held in immigration detention or removal centres (i.e. facilities detaining foreign national citizens for administrative purposes under immigration powers) were excluded. Studies that did not examine the impact of detention on mental health specifically were also excluded. Therefore any studies examining multi-morbidity involving both mental health disorders and physical disorders (e.g. infectious diseases) were excluded, as were studies focused on the attitudes and experiences of health professionals working within immigration detention. Reviews, grey literature, commentaries, conference abstracts, letters, editorials, books and short surveys were also excluded. We have however referred to relevant grey literature in the introduction and discussion sections.

Studies concerning child and adult detainees have been presented separately for the sake of clarity.

\section{Search strategy}

Relevant studies were identified through electronic searches of Embase (1980 to 2018 week 22), Ovid MEDLINE (1946 to May week 3 2018) and PsycINFO (1806 to May week 3 2018). Searches were limited to English language only. No other restrictions were applied to the searches. All search terms related to the following two main areas: mental health and immigration detention. The full electronic search strategy is presented in Additional file 1.

Electronic searches were supplemented by screening of reference lists of all included primary studies. In addition, the following journals published between 2013 and 2018 were hand searched: Forced Migrant Review, International Migration Review, International Migration, Journal of Refugee Studies, Journal of Traumatic Stress, Refuge, and Torture. Six International experts working in the field of forced migration and health (i.e. psychiatry, psychology and criminology) were contacted to nominate further papers eligible for inclusion. One response was received.

\section{Data extraction and quality appraisal}

Three reviewers (CM, RS, ZC) were involved in the process of independently screening titles and available abstracts of identified articles. If it was unclear whether a reference was relevant for the review, texts were retrieved in full. The same team of reviewers (CM, RS, ZC) independently assessed full texts to verify eligibility based on the above selection criteria. Disagreement was resolved either by discussion or with assistance of a third 
Table 1 Mental Health Problems in Immigration Detainees: Characteristics of 26 quantitative studies

\begin{tabular}{|c|c|c|c|c|c|c|}
\hline Author and year & Study Design & Sample & $\begin{array}{l}\text { Country of } \\
\text { Origin }\end{array}$ & $\begin{array}{l}\text { Country of } \\
\text { Study }\end{array}$ & Outcomes of interest & $\begin{array}{l}\text { Method of assessing } \\
\text { outcome }\end{array}$ \\
\hline Brabeck \& Xu [59] & $\begin{array}{l}\text { Cross sectional } \\
\text { survey }\end{array}$ & $\begin{array}{l}N=132 \text { immigrant } \\
\text { parents who accessed } \\
\text { immigrant community } \\
\text { organisations } \\
\text { Detention Duration: } \\
\text { unknown }\end{array}$ & $\begin{array}{l}\text { Various Latin } \\
\text { American } \\
\text { countries }\end{array}$ & $\begin{array}{l}\text { United } \\
\text { States }\end{array}$ & $\begin{array}{l}\text { Impact of detention } \\
\text { / deportation on } \\
\text { Latino immigrant } \\
\text { families }\end{array}$ & $\begin{array}{l}\text { Self-report survey informed } \\
\text { by Brabeck et al. [59] } \\
\text { Translated and back } \\
\text { translated }\end{array}$ \\
\hline $\begin{array}{l}\text { Cleveland \& } \\
\text { Rousseau [38] }\end{array}$ & $\begin{array}{l}\text { Cross-sectional, } \\
\text { comparison survey }\end{array}$ & $\begin{array}{l}N=122 \text { detained adult } \\
\text { asylum seekers, } N=66 \\
\text { non-detained adult } \\
\text { asylum seekers } \\
\text { Detention Duration: } \\
\text { mean } 31.2 \text { days, median } \\
17.5 \text { days }\end{array}$ & Unknown & Canada & $\begin{array}{l}\text { Anxiety, depression } \\
\text { \& PTSD }\end{array}$ & $\begin{array}{l}\text { HTQ, HSCL-25, modified } \\
\text { DEC }\end{array}$ \\
\hline Coffey et al. [40] & $\begin{array}{l}\text { Cross-sectional } \\
\text { semi-structured } \\
\text { interview and survey }\end{array}$ & $\begin{array}{l}N=17 \text { refugees } \\
\text { Detention Duration: } \\
\text { mean } 3 \text { years } 2 \text { months, } \\
\text { range } 1.6-4.5 \text { years }\end{array}$ & $\begin{array}{l}\text { Afghanistan, } \\
\text { Iraq, Iran and } \\
\text { neighbouring } \\
\text { countries }\end{array}$ & Australia & $\begin{array}{l}\text { Experience of } \\
\text { detention } \\
\text { PTSD, depression, } \\
\text { anxiety, quality of } \\
\text { life }\end{array}$ & $\begin{array}{l}\text { Semi-structured } \\
\text { interviews; HSCL-25, HTQ, } \\
\text { WHOQOL-Brief }\end{array}$ \\
\hline Cohen [50] & Data comparison & $\begin{array}{l}N=231 \text { self-harm } \\
\text { incident reports } \\
N=12 \text { suicide incident } \\
\text { reports from IRCs } \\
N=38 \text { suicides from } \\
\text { coroners and } \\
\text { ombudsman reports }\end{array}$ & Unknown & UK & $\begin{array}{l}\text { Rates of self-harm } \\
\text { and suicide }\end{array}$ & $\begin{array}{l}\text { Analysis of records, } \\
\text { coroner's files and } \\
\text { ombudsman's reports }\end{array}$ \\
\hline Cwikel et al. [37] & $\begin{array}{l}\text { Cross-sectional, } \\
\text { comparison survey }\end{array}$ & $\begin{array}{l}N=47 \text { female brothel } \\
\text { workers in detention, } \\
N=55 \text { female brothel } \\
\text { workers } \\
\text { Detention Duration: } \\
\text { unknown }\end{array}$ & $\begin{array}{l}\text { Former Soviet } \\
\text { Union }\end{array}$ & Israel & $\begin{array}{l}\text { PTSD, depression, } \\
\text { somatic symptoms, } \\
\text { suicidality }\end{array}$ & $\begin{array}{l}\text { PCL, CES-D, constructed } \\
\text { scale of physical } \\
\text { symptoms, constructed } \\
\text { scale of both past trauma } \\
\text { and work trauma } \\
\text { Russian speaking } \\
\text { psychiatrist }\end{array}$ \\
\hline Graf et al. [47] & $\begin{array}{l}\text { Cross-sectional } \\
\text { survey }\end{array}$ & $\begin{array}{l}N=80 \text { males detained } \\
\text { for violation of the Swiss } \\
\text { Aliens Act } \\
\text { Detention Duration: } 4 \\
\text { days }\end{array}$ & $\begin{array}{l}31 \text { different } \\
\text { countries }\end{array}$ & Switzerland & $\begin{array}{l}\text { Prevalence rates of } \\
\text { mental health } \\
\text { disorders }\end{array}$ & $\begin{array}{l}\text { BJMHS, CIDI (clinical } \\
\text { psychologist), SCL-90R, } \\
\text { subjective mental health } \\
\text { questionnaire within } 4 \\
\text { days of detention, SCL- } \\
90 \text { R and self-report again } \\
6 \text { months later where } \\
\text { possible } \\
\text { Material professionally } \\
\text { translated into several } \\
\text { languages }\end{array}$ \\
\hline $\begin{array}{l}\text { Green \& Eager } \\
\text { [36] }\end{array}$ & $\begin{array}{l}\text { Cross-sectional } \\
\text { analysis of health } \\
\text { records }\end{array}$ & $\begin{array}{l}N=720 \\
\text { Detention Duration: } \\
\text { range } 3-24 \text { months }\end{array}$ & $\begin{array}{l}58 \text { different } \\
\text { countries }\end{array}$ & Australia & $\begin{array}{l}\text { Estimated incidence } \\
\text { rates of new health } \\
\text { conditions, new } \\
\text { mental health } \\
\text { conditions and new } \\
\text { injuries for each } \\
\text { cohort }\end{array}$ & $\begin{array}{l}\text { Health records coded by } \\
\text { clinical coder }\end{array}$ \\
\hline Hedrick [49] & $\begin{array}{l}\text { Analysis of incident } \\
\text { reports }\end{array}$ & Unknown & Unknown & Australia & Self-harm & Self-harm incident reports \\
\hline $\begin{array}{l}\text { Ichikawa et al. } \\
\text { [41] }\end{array}$ & $\begin{array}{l}\text { Cross-sectional, } \\
\text { comparison survey }\end{array}$ & $\begin{array}{l}N=18 \text { former detained } \\
\text { asylum seekers, } N=37 \\
\text { non-detained asylum } \\
\text { seekers } \\
\text { Accessible through a } \\
\text { group of voluntary } \\
\text { lawyers representing } \\
\text { them and two NGO's } \\
\text { Detention Duration: } \\
\text { median } 7 \text { months, range } \\
4-10 \text { months }\end{array}$ & Afghanistan & Japan & $\begin{array}{l}\text { Anxiety, depression, } \\
\text { PTSD }\end{array}$ & $\begin{array}{l}\text { HSCL-25, HTQ } \\
\text { Translated into Dari and } \\
\text { read out to participants } \\
\text { by NGO caseworkers }\end{array}$ \\
\hline
\end{tabular}


Table 1 Mental Health Problems in Immigration Detainees: Characteristics of 26 quantitative studies (Continued)

\begin{tabular}{|c|c|c|c|c|c|c|}
\hline Author and year & Study Design & Sample & $\begin{array}{l}\text { Country of } \\
\text { Origin }\end{array}$ & $\begin{array}{l}\text { Country of } \\
\text { Study }\end{array}$ & Outcomes of interest & $\begin{array}{l}\text { Method of assessing } \\
\text { outcome }\end{array}$ \\
\hline $\begin{array}{l}\text { Keller, Rosenfeld } \\
\text { et al. [44] }\end{array}$ & $\begin{array}{l}\text { Cross-sectional } \\
\text { survey }\end{array}$ & $\begin{array}{l}N=70 \text { detained asylum } \\
\text { seekers } \\
\text { Detention Duration: } \\
\text { median } 7 \text { months, range } \\
2-42 \text { months }\end{array}$ & $\begin{array}{l}\text { Multiple } \\
\text { countries }\end{array}$ & USA & $\begin{array}{l}\text { Anxiety, depression, } \\
\text { PTSD }\end{array}$ & $\begin{array}{l}\text { HSCL-25, HTQ (+ two } \\
\text { month follow up) } \\
\text { Scales translated by } \\
\text { interpreter, interviewed } \\
\text { by experienced physician }\end{array}$ \\
\hline Lorek et al. [52] & $\begin{array}{l}\text { Cross-sectional } \\
\text { survey }\end{array}$ & $\begin{array}{l}N=11 \text { detained children, } \\
N=9 \text { parents who } \\
\text { responded to free legal } \\
\text { assistance } \\
\text { Detention Duration: } \\
\text { range } 11-115 \text { days }\end{array}$ & Multiple & Britain & $\begin{array}{l}\text { Mental and physical } \\
\text { health of children } \\
\text { held within } \\
\text { immigration } \\
\text { detention centre }\end{array}$ & $\begin{array}{l}\text { Clinical diagnostic } \\
\text { interviews, SCAS, DSRS, R- } \\
\text { IES-13, SDQ, CORE, } \\
\text { observations } \\
\text { Psychologist and } \\
\text { paediatricians had all } \\
\text { been trained in carrying } \\
\text { out cross-cultural } \\
\text { assessments and worked } \\
\text { regularly with asylum } \\
\text { seeking children and } \\
\text { families }\end{array}$ \\
\hline Mares [54] & $\begin{array}{l}\text { Secondary analysis } \\
\text { of Australian Human } \\
\text { Rights Commissions } \\
\text { Data Set }\end{array}$ & $\begin{array}{l}N=131 \text { adults and } N= \\
35 \text { completed } \mathrm{K} 10 \\
\mathrm{~N}=70 \text { completed SDQ } \\
\text { Detention Duration: } \\
\text { mean } 8 \text { months, range } \\
\text { 90-390 days }\end{array}$ & Unknown & Australia & $\begin{array}{l}\text { Psychological } \\
\text { distress, behavioural } \\
\text { difficulties }\end{array}$ & K10, SDQ \\
\hline $\begin{array}{l}\text { Mares \& Jureidini } \\
\text { [53] }\end{array}$ & $\begin{array}{l}\text { Assessment of } \\
\text { referrals into CAMHS }\end{array}$ & $\begin{array}{l}N=16 \text { adults, } 20 \\
\text { children, } 10 \text { families } \\
\text { Detention Duration: } \\
\text { mean } 1 \text { year } 3 \text { months, } \\
\text { range } 12-18 \text { months }\end{array}$ & $\begin{array}{l}\text { Iran, Iraq, } \\
\text { Afghanistan, } \\
\text { Palestine }\end{array}$ & Australia & Clinical assessment & $\begin{array}{l}\text { Clinical instrument } \\
\text { unknown }\end{array}$ \\
\hline $\begin{array}{l}\text { Momartin et al. } \\
\text { [51] }\end{array}$ & $\begin{array}{l}\text { Cross-sectional } \\
\text { survey }\end{array}$ & $\begin{array}{l}N=49 \text { former } \\
\text { immigration detainees on } \\
\text { temporary protection visas } \\
\text { and } N=67 \text { granted } \\
\text { permanent protection visas } \\
\text { Detention duration: mean } \\
12 \text { months }\end{array}$ & Unknown & Australia & $\begin{array}{l}\text { PTSD, anxiety, } \\
\text { depression, general } \\
\text { health, living } \\
\text { difficulties and } \\
\text { experiences of } \\
\text { detention }\end{array}$ & $\begin{array}{l}\text { HTQ, HSCL-25, GHQ-30, } \\
\text { MOSSF-12, PMDC, DEC }\end{array}$ \\
\hline $\begin{array}{l}\text { Puthoopparambil } \\
\text { et al. [46] }\end{array}$ & $\begin{array}{l}\text { Cross-sectional } \\
\text { survey }\end{array}$ & $\begin{array}{l}N=127 \text { immigration } \\
\text { detainees } \\
\text { Detention Duration: } \\
\text { mean } 37.8 \text { days }\end{array}$ & $\begin{array}{l}46 \text { different } \\
\text { countries }\end{array}$ & Sweden & Quality of life & $\begin{array}{l}\text { WHOQOL-BREF - incl. Six } \\
\text { psychological questions } \\
\text { Authorised telephone } \\
\text { interpreters (used by } 77 \\
\text { participants) }\end{array}$ \\
\hline Robjant et al. [11] & $\begin{array}{l}\text { Cross-sectional, } \\
\text { comparison survey }\end{array}$ & $\begin{array}{l}\mathrm{N}=67 \text { detained asylum } \\
\text { seekers, } N=30 \text { detained } \\
\text { former prisoners, } \mathrm{N}=49 \\
\text { asylum seekers living in } \\
\text { the community } \\
\text { Detention Duration: } \\
\text { unknown }\end{array}$ & Unknown & UK & $\begin{array}{l}\text { PTSD, depression, } \\
\text { anxiety }\end{array}$ & $\begin{array}{l}\text { HADS, IES-R, PDS } \\
\text { English }\end{array}$ \\
\hline Rojas-Flores [57] & $\begin{array}{l}\text { Cross sectional } \\
\text { comparison survey }\end{array}$ & $\begin{array}{l}N=39 \text { children of } \\
\text { detained or deported } \\
\text { parents } \\
N=42 \text { unauthorised no } \\
\text { history of detention or } \\
\text { deportation } \\
N=16 \text { Legal permanent } \\
\text { resident } \\
\text { Detention Duration: } \\
\text { unknown }\end{array}$ & $\begin{array}{l}\text { Mexico or } \\
\text { Central } \\
\text { America }\end{array}$ & US & $\begin{array}{l}\text { PTSD, depression, } \\
\text { behavioural } \\
\text { difficulties, daily } \\
\text { functioning }\end{array}$ & $\begin{array}{l}\text { UCLA PTSD-RI, CES-DSC, } \\
\text { BASC-2 PRS-C, TSCYC-SP, } \\
\text { BASC-2 TRS-C, CAFAS }\end{array}$ \\
\hline $\begin{array}{l}\text { Rothe et al. } \\
\text { (2002a) }\end{array}$ & $\begin{array}{l}\text { Cross sectional } \\
\text { survey }\end{array}$ & $\begin{array}{l}N=74 \text { adolescents } \\
\text { Detention Duration: 4-6 } \\
\text { months }\end{array}$ & Cuba & US & $\begin{array}{l}\text { PTSD, psychological } \\
\text { distress }\end{array}$ & $\begin{array}{l}\text { PTSDRI, checklist of PTSD } \\
\text { symptoms }\end{array}$ \\
\hline $\begin{array}{l}\text { Rothe et al. } \\
\text { (2002b) }\end{array}$ & $\begin{array}{l}\text { Cross sectional } \\
\text { survey }\end{array}$ & $\begin{array}{l}N=87 \text { adolescents } \\
\text { Detention Duration: }\end{array}$ & Cuba & US & $\begin{array}{l}\text { PTSD, behavioural } \\
\text { difficulties }\end{array}$ & PTSDRI, CBCL-TRF \\
\hline
\end{tabular}


Table 1 Mental Health Problems in Immigration Detainees: Characteristics of 26 quantitative studies (Continued)

\begin{tabular}{|c|c|c|c|c|c|c|}
\hline Author and year & Study Design & Sample & $\begin{array}{l}\text { Country of } \\
\text { Origin }\end{array}$ & $\begin{array}{l}\text { Country of } \\
\text { Study }\end{array}$ & Outcomes of interest & $\begin{array}{l}\text { Method of assessing } \\
\text { outcome }\end{array}$ \\
\hline & & mean 6-8 months & & & & \\
\hline Sen et al. [45] & $\begin{array}{l}\text { Observational, } \\
\text { Cross-sectional }\end{array}$ & $\begin{array}{l}N=101 \text { male detained in } \\
\text { immigration removal } \\
\text { centre } \\
\text { Detention Duration: } \\
\text { unknown }\end{array}$ & $\begin{array}{l}27 \text { different } \\
\text { countries }\end{array}$ & UK & Neurodevelopmental & $\begin{array}{l}\text { MINI v6, SAPAS, AQ-10, } \\
\text { ASRS, LDSQ, CANFOR } \\
\text { No interpreters }\end{array}$ \\
\hline $\begin{array}{l}\text { Sobhanian et al. } \\
\text { [42] }\end{array}$ & $\begin{array}{l}\text { Cross-sectional } \\
\text { survery }\end{array}$ & $\begin{array}{l}N=150 \text { former refugee } \\
\text { detainees } \\
\text { Detention Duration: } \\
\text { mean } 11.3 \text { months, range } \\
2-21 \text { months }\end{array}$ & $\begin{array}{l}\text { Iran, } \\
\text { Afghanistan }\end{array}$ & Australia & $\begin{array}{l}\text { Psychological status } \\
\text { and quality of life }\end{array}$ & $\begin{array}{l}\text { T-FAST, QOLI, POMS, SIS } \\
\text { Translated and back } \\
\text { translated into Farsi, self } \\
\text { report administered } \\
\text { under supervision of } \\
\text { clinical psychologist }\end{array}$ \\
\hline Steel et al. [23] & Cross-sectional & $\begin{array}{l}\mathrm{N}=10 \text { families ( } 14 \text { adults, } \\
20 \text { children) held for in } \\
\text { immigration detention } \\
\text { for more than two years } \\
\text { Detention Duration: } \\
\text { mean } 2 \text { years } 4 \text { months, } \\
\text { range } 2 \text { years } 2 \text { months - } \\
2 \text { years } 8 \text { months }\end{array}$ & Unknown & Australia & Psychiatric Status & $\begin{array}{l}\text { DEC, DSC, K-SADS-PL, } \\
\text { SCID-IV, Parenting } \\
\text { Questionnaire, } \\
\text { Over the telephone by } \\
\text { same language-speaking } \\
\text { psychologists with prior } \\
\text { professional experience } \\
\text { working with refugees }\end{array}$ \\
\hline Steel et al. [43] & $\begin{array}{l}\text { Cross-sectional, } \\
\text { comparison survey }\end{array}$ & $\begin{array}{l}N=241 \text { Arabic-speaking } \\
\text { Mandaean refugees } \\
\text { Detention Duration: } \\
\text { median } 6 \text { months }\end{array}$ & $\begin{array}{l}\text { Mainly Iran and } \\
\text { Iraq }\end{array}$ & Australia & $\begin{array}{l}\text { Anxiety, depression } \\
\text { \& PTSD }\end{array}$ & $\begin{array}{l}\text { HTQ, HSCL-25, MOSSF-12, } \\
\text { PMLD, DEC, DSC } \\
\text { Translated and back } \\
\text { translated by certified } \\
\text { Arabic-speaking } \\
\text { healthcare interpreter }\end{array}$ \\
\hline Steel et al. [39] & Longitudinal survey & $\begin{array}{l}\mathrm{N}=47 \text { former } \\
\text { immigration detainees on } \\
\text { temporary protection } \\
\text { visas and } N=57 \text { granted } \\
\text { permanent protection } \\
\text { visas attending a state- } \\
\text { wide early intervention } \\
\text { program in New South } \\
\text { Wales } \\
\text { Detention Duration: } \\
\text { meadian } 8 \text { months, range } \\
1-30 \text { months }\end{array}$ & $\begin{array}{l}\text { Iran, } \\
\text { Afghanistan }\end{array}$ & Australia & $\begin{array}{l}\text { Anxiety, depression } \\
\text { and PTSD }\end{array}$ & $\begin{array}{l}\text { HTQ, HSCL-25, GHQ-30, } \\
\text { PSWQ, PMLD } \\
\text { Baseline and 2-year follow } \\
\text { up } \\
\text { Measures translated by } \\
\text { experienced clinical } \\
\text { psychologist fluent in } \\
\text { both dialects, and back } \\
\text { translated. Interviews } \\
\text { undertaken by Fasi / Dari } \\
\text { speaking psychologists }\end{array}$ \\
\hline $\begin{array}{l}\text { Young \& Gordon } \\
{[48]}\end{array}$ & $\begin{array}{l}\text { Secondary analysis } \\
\text { of Australian Human } \\
\text { Rights Commissions } \\
\text { Data Set }\end{array}$ & $\begin{array}{l}N=1354 \text { detained } \\
\text { refugees, asylum seekers } \\
\text { and 'others' }\end{array}$ & Unknown & Australia & PTSD, mental health & $\begin{array}{l}\text { K10, HTQ, HoNOS, } \\
\text { HoNOSCA }\end{array}$ \\
\hline Zwi et al. [58] & $\begin{array}{l}\text { Cross sectional } \\
\text { survey }\end{array}$ & $\begin{array}{l}N=48 \text { detained children } \\
N=38 \text { child asylum } \\
\text { seekers in community }\end{array}$ & $\begin{array}{l}\text { Eastern } \\
\text { Mediterranean, } \\
\text { South East } \\
\text { Asia, Pacific, } \\
\text { Africa, Stateless } \\
\text { origin }\end{array}$ & Australia & $\begin{array}{l}\text { Behavioural } \\
\text { difficulties }\end{array}$ & $\mathrm{SDQ}$ \\
\hline
\end{tabular}

ASRS Adult ADHD Self-Report Scales, AQ-10 Autism-Spectrum Quotient 10, BASC-2 PRS-C Behaviour Assessment System for Children-2nd Edition, Parent Rating Scales-Child, BASC-2 TRS-C Behaviour Assessment System for Children-2nd Edition, Teacher Rating Scales-Child, BJMHS Brief Jail Mental Health Screen, CAFAS Child and Adolescent Functional Assessment Scale, CAMHS Child and Adolescent Mental Health Services, CANFOR Camberwell Assessment of Needs - Forensic Version, CBCL-TRF Child Behavioral Check List - Teachers Report Form, CES-D Center for Epidemiologic Studies Depression, CES-DSC Centre for Epidemiologic Studies Depression Scale for Children, CIDI Composite International Diagnostic Interview, CORE Clinical Outcomes in Routine Evaluation, DEC Detention Experience Checklist, DSC Detention Symptom Checklist, DSRS Birleson Depression Self-Rating Scale, GHQ-30 General Health Questionnaire 30, HADS Hospital Anxiety Depression Scale, HSCL-25 Hopkins Symptoms Checklist 25, HoNOS Health of Nation Outcomes Scale, HoNOSCA Health of Nation Outcomes Scale for Children and Adolescents, HTQ Harvard Trauma Questionnaire, IES-R Impact of Events Scale Revised, K10 Kessler 10, K-SADS-PL Schedule for Affective Disorders and Schizophrenia for School-Aged Children-Present and Life Time Version, LDSQ Learning Disability Screening Questionnaire, MOSSF-12Medical Outcomes Study Short Form, MINI v6 Mini International Neuropsychiatric Interview v6.0, NGO Non-governmental Organisation, PCL PTSD checklist, PDS Posttraumatic diagnostic scale, PMDC Post Migration Difficulties Checklist, PMLD - Post-migration living difficulties and detention experiences checklist, POMS Profile of Mood States, PSWQ Penn State Worry Questionnaire, PTSDRI Posttraumatic Stress Disorder Reaction Index, QOLI Quality of Life Inventory, R-IES-13 Revised Impact of Events Scale-13, SAPAS Standardized Assessment of Personality Abbreviated Scale, SCAS Spence Children's Anxiety Scale, SCID-IV Structured Clinical Interview for DSM-IV Axis I Disorders, SCL-90-R Symptoms Checklist-90-R, SDQ Strengths and Difficulties Questionnaire, SIS Suicide Ideation Scale, T-FAST Truncated Firestone Assessment of Self destructive Thoughts, TSCYC-SP Trauma Symptom Checklist for Young Children - Spanish Version, UCLA PTSD-RI UCLA Posttraumatic Stress Disorder Reaction Index, WHOQOL-Brief World Health Organisation Quality of Life Short Version 
reviewer (CK, KR or MW). Data from all included studies was double extracted by a team of four reviewers $(\mathrm{CM}$, MW, RS, ZC) into a standardised electronic spreadsheet. The following information was extracted from each study: first author, year of publication, population sampled, country where study was carried out, study design, measures used to assess mental health or psychological domains of quality of life, outcomes and length of immigration detention. The quality of studies which met the inclusion criteria was appraised independently by two from a team of three reviewers (CK, CM, MW) using a modified version of the qualitative Critical Appraisal Skills Programme Checklist [35].

Each item was rated as high (2), intermediate (1) or low (0) quality, resulting in a maximum potential total score of 26 (see Table 2 for breakdown of scores). Discrepancies in scores between reviewers were resolved by discussion.

\section{Results}

The study selection process is outlined in Fig. 1. Twenty-six relevant studies reporting on a total of 2099 participants were included in the review. Of these, 16 studies reported on adults, 9 studies on children and families and one reported on both. Studies were conducted in Australia, Canada, Israel, Japan, Sweden, Switzerland, the UK and the US. Not all studies listed individual countries of origin, yet as many as 58 countries were represented within a single study [36]. Key characteristics of all included studies can be found in Table 2 .

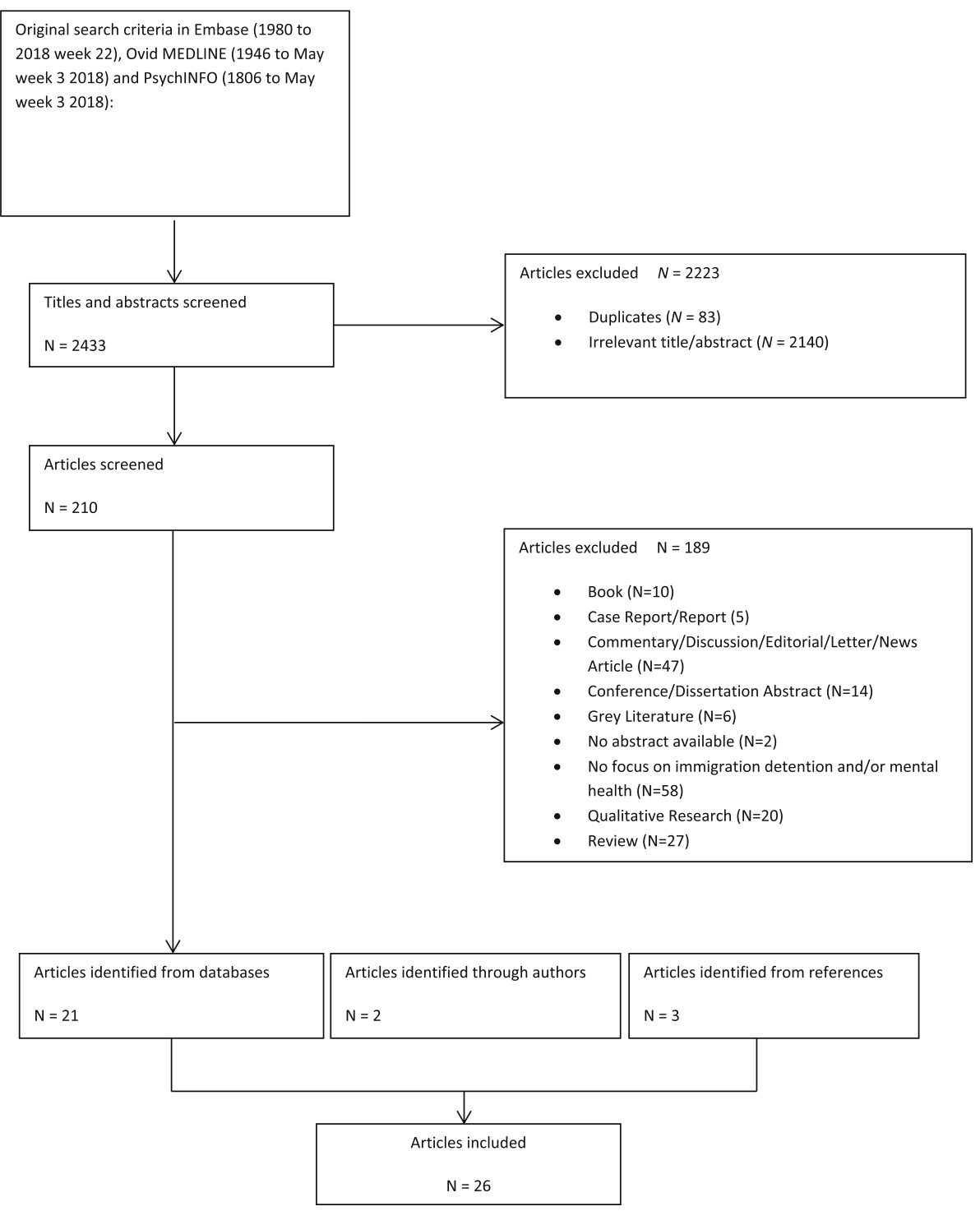

Fig. 1 Study flow chart 
Table 2 Quality Review of 26 studies

\begin{tabular}{|c|c|c|c|c|c|c|c|}
\hline \multicolumn{8}{|c|}{ Quality Question 1-7 } \\
\hline Study & $\begin{array}{l}\text { Clear focus on } \\
\text { question }\end{array}$ & $\begin{array}{l}\text { Appropriate } \\
\text { design }\end{array}$ & $\begin{array}{l}\text { Appropriate } \\
\text { sampling } \\
\text { method }\end{array}$ & $\begin{array}{l}\text { Appropriate } \\
\text { sample }\end{array}$ & $\begin{array}{l}\text { Tolerable } \\
\text { level of non- } \\
\text { participation }\end{array}$ & $\begin{array}{l}\text { Exposure } \\
\text { (detention) } \\
\text { appropriately } \\
\text { addressed }\end{array}$ & $\begin{array}{l}\text { Outcomes (mental health } \\
\text { symptoms and disorders) } \\
\text { appropriately addressed }\end{array}$ \\
\hline Brabeck \& Xu [59] & High & High & High & Intermediate & Intermediate & Intermediate & Low \\
\hline $\begin{array}{l}\text { Cleveland \& } \\
\text { Rousseau [38] }\end{array}$ & High & High & Intermediate & High & Intermediate & High & High \\
\hline Coffey et al. [40] & High & High & Intermediate & Intermediate & Intermediate & High & High \\
\hline Cohen [50] & Intermediate & Low & Low & Low & Low & Intermediate & Intermediate \\
\hline Cwikel et al. [37] & High & High & Low & Intermediate & Low & High & High \\
\hline Graf et al. [47] & High & High & High & Intermediate & Low & High & High \\
\hline $\begin{array}{l}\text { Green \& Eagar } \\
{[36]}\end{array}$ & High & Intermediate & High & Intermediate & High & High & Intermediate \\
\hline Hedrick [49] & High & High & High & Intermediate & High & Intermediate & Intermediate \\
\hline $\begin{array}{l}\text { Ichikawa et al. } \\
\text { [41] }\end{array}$ & High & High & High & High & High & High & High \\
\hline Keller et al. [44] & Intermediate & High & Intermediate & High & High & High & High \\
\hline Lorek et al. [52] & High & High & Intermediate & Intermediate & Low & High & Intermediate \\
\hline Mares [54] & High & Intermediate & Intermediate & Intermediate & Low & High & High \\
\hline $\begin{array}{l}\text { Mares \& Jureidini } \\
{[53]}\end{array}$ & High & High & Intermediate & Intermediate & Intermediate & High & Intermediate \\
\hline $\begin{array}{l}\text { Momartin et al. } \\
\text { [51] }\end{array}$ & High & High & Intermediate & Intermediate & High & High & High \\
\hline $\begin{array}{l}\text { Puthoopparambil } \\
\text { et al. (2009) }\end{array}$ & High & High & High & Intermediate & Intermediate & High & High \\
\hline Robjant et al. [11] & High & High & Intermediate & High & High & High & High \\
\hline $\begin{array}{l}\text { Rojas-Flores, L. } \\
{[57]}\end{array}$ & High & High & Intermediate & Intermediate & Low & Intermediate & High \\
\hline Rothe et al. [55] & Intermediate & High & Intermediate & Low & Low & High & Intermediate \\
\hline Rothe et al. [56] & High & High & Low & Low & Low & High & High \\
\hline Sen et al. [45] & High & High & Intermediate & Intermediate & Intermediate & High & High \\
\hline $\begin{array}{l}\text { Sobhanian et al. } \\
\text { [42] }\end{array}$ & High & Intermediate & Low & Intermediate & Low & High & High \\
\hline Steel et al. [23] & High & High & High & Intermediate & High & High & Intermediate \\
\hline Steel et al. [43] & High & High & High & Intermediate & High & High & High \\
\hline Steel et al. (2011) & High & High & High & High & High & High & High \\
\hline $\begin{array}{l}\text { Young \& Gordon } \\
\text { [48] }\end{array}$ & High & High & High & Intermediate & Intermediate & Intermediate & High \\
\hline Zwi et al. [58] & High & High & Intermediate & High & Intermediate & High & High \\
\hline \multicolumn{8}{|c|}{ Quality Question 8-13 } \\
\hline Study & $\begin{array}{l}\text { Consideration } \\
\text { of cofounders }\end{array}$ & $\begin{array}{l}\text { Appropriate } \\
\text { conduction of } \\
\text { statistical } \\
\text { analyses }\end{array}$ & $\begin{array}{l}\text { Reporting of } \\
\text { confidence } \\
\text { intervals }\end{array}$ & $\begin{array}{l}\text { Consideration } \\
\text { of ethical } \\
\text { issues }\end{array}$ & $\begin{array}{l}\text { Adequate } \\
\text { support for } \\
\text { conclusions }\end{array}$ & $\begin{array}{l}\text { Generalisability } \\
\text { of findings }\end{array}$ & Total Score \\
\hline Brabeck \& Xu [59] & High & High & High & Intermediate & High & Intermediate & $19 / 26$ \\
\hline $\begin{array}{l}\text { Cleveland \& } \\
\text { Rousseau [38] }\end{array}$ & High & High & High & High & High & High & $24 / 26$ \\
\hline Coffey et al. [40] & Low & High & High & High & High & Intermediate & $20 / 26$ \\
\hline Cohen [50] & Low & Low & Low & Intermediate & High & Intermediate & $7 / 26$ \\
\hline Cwikel et al. [37] & High & High & Intermediate & Intermediate & High & Intermediate & $18 / 26$ \\
\hline
\end{tabular}


Table 2 Quality Review of 26 studies (Continued)

\begin{tabular}{|c|c|c|c|c|c|c|c|}
\hline Graf et al. [47] & Low & High & Low & High & High & High & $19 / 26$ \\
\hline $\begin{array}{l}\text { Green \& Eagar } \\
\text { [36] }\end{array}$ & High & High & High & Intermediate & High & High & $22 / 26$ \\
\hline Hedrick [49] & Intermediate & High & Low & Intermediate & High & High & $19 / 26$ \\
\hline $\begin{array}{l}\text { Ichikawa et al. } \\
\text { [41] }\end{array}$ & High & High & High & High & High & Intermediate & $25 / 26$ \\
\hline Keller et al. [44] & High & High & High & High & High & High & $23 / 26$ \\
\hline Lorek et al. [52] & Intermediate & Low & Low & Intermediate & High & Intermediate & $14 / 26$ \\
\hline Mares [54] & Low & High & Low & High & High & Intermediate & $16 / 26$ \\
\hline $\begin{array}{l}\text { Mares \& Jureidini } \\
{[53]}\end{array}$ & Low & Intermediate & Low & Low & High & Intermediate & $14 / 26$ \\
\hline $\begin{array}{l}\text { Momartin et al. } \\
\text { [51] }\end{array}$ & High & High & High & Intermediate & High & Intermediate & $22 / 26$ \\
\hline $\begin{array}{l}\text { Puthoopparambil } \\
\text { et al. [46] }\end{array}$ & High & High & High & High & High & High & $24 / 26$ \\
\hline Robjant et al. [11] & High & High & High & High & High & High & $25 / 26$ \\
\hline $\begin{array}{l}\text { Rojas-Flores, L. } \\
\text { [57] }\end{array}$ & High & High & Intermediate & Intermediate & High & Intermediate & $18 / 26$ \\
\hline $\begin{array}{l}\text { Rothe et al. } \\
\text { (2002a) }\end{array}$ & Low & Low & Low & Low & High & Low & $9 / 26$ \\
\hline $\begin{array}{l}\text { Rothe et al. } \\
\text { (2002b) }\end{array}$ & High & High & Low & Intermediate & High & Low & $15 / 26$ \\
\hline Sen et al. [45] & Intermediate & High & Low & High & High & High & $19 / 26$ \\
\hline $\begin{array}{l}\text { Sobhanian et al. } \\
\text { [42] }\end{array}$ & High & High & Low & Intermediate & High & High & $15 / 26$ \\
\hline Steel et al. [23] & Intermediate & Intermediate & Low & High & High & Intermediate & $19 / 26$ \\
\hline Steel et al. [43] & High & High & Intermediate & High & High & Intermediate & $23 / 26$ \\
\hline Steel et al. [39] & Intermediate & Intermediate & High & High & High & Intermediate & $24 / 26$ \\
\hline $\begin{array}{l}\text { Young \& Gordon } \\
\text { [48] }\end{array}$ & Low & High & Low & Intermediate & High & Intermediate & $17 / 26$ \\
\hline Zwi et al. [58] & Intermediate & High & High & Intermediate & High & High & $22 / 26$ \\
\hline
\end{tabular}

\section{Adults}

A total of 17 studies reported data on the mental health consequences of immigration detention in adults. These studies involved a total of 1168 participants (Note: this number only includes studies in which primary data was collected). Three studies on parental health are discussed separately in the 'Child and Families' subsection below. Six studies were conducted as comparison studies, with a total of 331 non-detained controls. Ten studies were conducted during detention, six were conducted following detention, and one included both current and past detainees. Reasons given for detention varied widely and were not consistently reported. Many were asylum seekers and some studies also included foreign national prisoners or ex-prisoners awaiting deportation, illegal workers and undocumented entrants. Participant ages ranged from 15 to 80 years of age. Approximately two thirds of all participants were male. One study focused exclusively on females, who were illegal sex workers detained in Israel and awaiting deportation [37]. Studies differed widely in the participants' duration of detention. Some studies reported average duration in terms of the mean, others reported median durations. For those reporting mean durations, these ranged between 4 days to 3.2 years. Reported median durations ranged between 6 to 8 months. Reported ranges fell between 2- to 4 months. Various pre-migratory traumatic events were reported, including but not limited to torture. Where reported, subjection to torture ranged from 12.2-100\%.

Overall, 11 studies employed self-report measures, one used self-report measures along with clinical assessment, one used clinical assessments only and four analysed health records. These studies are reviewed below in terms of method of measuring mental health outcomes (self-report and clinical assessments), use of a control design, detention duration and release, and gender.

\section{Self-report (see Table 3)}

Self-report data (predominantly using clinically validated rating scales) relating to detention experiences 
Table 3 Assessment and Prevalence Rates of Mental Health Difficulties in currently detained and formerly detained Adults

\begin{tabular}{|c|c|c|c|c|c|c|c|c|c|}
\hline Mental Health Difficulty & $\begin{array}{l}\text { Cleveland \& } \\
\text { Rousseau [38] }\end{array}$ & $\begin{array}{l}\text { Coffey et } \\
\text { al. [40] }\end{array}$ & $\begin{array}{l}\text { Cwikel et } \\
\text { al. [37] }\end{array}$ & $\begin{array}{l}\text { Ichikawa et } \\
\text { al. [41] }\end{array}$ & $\begin{array}{l}\text { Keller et } \\
\text { al. [44] }\end{array}$ & $\begin{array}{l}\text { Robjant et } \\
\text { al. [11] }\end{array}$ & $\begin{array}{l}\text { Sen et al. } \\
{[45]}\end{array}$ & $\begin{array}{l}\text { Steel et } \\
\text { al. [43] }\end{array}$ & $\begin{array}{l}\text { Steel et } \\
\text { al. [39] }\end{array}$ \\
\hline Depression & $\sqrt{ }$ & $88 \%$ & $79 \%$ & $\sqrt{ }$ & $86 \%$ & $\sqrt{ }$ & $52.5 \%$ & $\sqrt{ }$ & $\sqrt{ }$ \\
\hline Anxiety & $\sqrt{ }$ & - & - & $\sqrt{ }$ & $77 \%$ & $72 \%$ & $12 \%$ & $\sqrt{ }$ & $\sqrt{ }$ \\
\hline PTSD & $\sqrt{ }$ & $70 \%$ & $17 \%$ & $\sqrt{ }$ & $50 \%$ & $76 \%$ & $20.8 \%$ & $\sqrt{ }$ & $\sqrt{ }$ \\
\hline Specific Phobia & - & - & - & - & - & & & & - \\
\hline $\begin{array}{l}\text { Psychotic or Depressive } \\
\text { Affective Disorder }\end{array}$ & - & - & - & - & - & - & $10 \%$ & - & - \\
\hline Personality Disorder & - & - & - & - & - & - & $34.7 \%$ & - & - \\
\hline Suicidal Ideation & - & - & $47 \%$ & - & $26 \%$ & - & - & - & - \\
\hline Suicide Attempt & - & - & $19 \%$ & - & $3 \%$ & - & - & - & - \\
\hline Somatisation & - & - & $40-60 \%$ & - & - & - & - & - & - \\
\hline Autism & - & - & - & - & - & - & $15 \%$ & - & - \\
\hline ADHD & - & - & - & - & - & - & $14 \%$ & - & - \\
\hline OCD & - & - & - & - & - & - & $9 \%$ & - & - \\
\hline
\end{tabular}

consistently identify severe levels of mental health problems amongst detainees. Most studies $(N=9)$ reported on symptoms of anxiety, depression and PTSD, which appear to be the most common forms of mental health difficulties experienced by detainees both during [36-39] and following detention (see below; [40-43]. Whilst not differentiating between types of traumatic experiences, greater trauma exposure prior to detention was significantly associated with higher rates of such symptoms [37, 38, 41]. Gaining temporary protection rather than permanent protection was an independent contributor to the risk of on-going depression and PTSD [43]. Isolation from family members and living alone were also associated with higher rates of such symptoms [41, 43].

Somatization, suicidal ideation, substance use, personality disorder and neurodevelopment disorders were also reported in some studies [37, 44, 45].

Three studies measured the quality of life of detainees and uniformly reported low quality of life ratings, particularly across psychological domains [40, 42, 46]. One of these studies found that levels of quality of life were mediated by the level of perceived support and satisfaction with the care received from detention staff [46].

\section{Clinical assessments}

Two of the identified studies involved diagnostic clinical assessments. Both of these were restricted to male detainees. Graf and colleagues [47], using the WHO Composite International Diagnostic Interview (CIDI), found a $76 \%$ point prevalence of mental health disorders in detainees, with $26.2 \%$ meeting criteria for serious mental illness within 4 days of admission. The most common disorders were found to be affective disorders (36\%), anxiety disorders (34\%) and PTSD (23\%). Similar prevalence rates were found in a study by Sen et al. [45], which combined self-report measures with clinical assessments. They found the prevalence of any mental health, developmental or personality disorder to be $74.3 \%$, with over half of the sample screened positive for more than one disorder. Seventy-three percent of the sample reported unmet psychological needs.

\section{Secondary health data}

Four studies analysed pre-existing health data obtained from detention centres. Data recording was poor across most studies, with authors facing flawed and unsystematic documentation. One study drew on data obtained from health records within detention centres [36]. Despite the reported lack of available records spanning the entire detention period of many detainees, the findings suggest that mental health issues were identified in $6 \%$ of detainees. The reason for being detained was also found to contribute to new mental health problems, with unauthorised boat arrivals displaying the highest rates of psychiatric morbidity (27\%). Asylum seekers had more health problems including mental health problems than other detainees. Only $7 \%$ of all recorded detention centre interventions included formal mental state examinations. A further study reported much higher numbers, with approximately half the detainees suffering from PTSD symptoms [48]. Two studies looked at rates of suicide and/or self-harm using data extracted from incident reports within detention centres in Australia [49] and the UK [50]. Self-harm was estimated to occur in $22 \%$ (including attempted hanging) and $12.8 \%$ respectively. Where provided, precipitating factors to self-harm revolved around detention conditions and procedural 
related factors (such as duration of claims) and negative decisions [49]. Suicides in immigration detention were estimated at 222 per 100,000 between 2002 and 2004, with the majority of deaths being caused by hanging [50]. Of the deaths in detainees reported in coroner's reports and Prisoner's Ombudsman's reports, the majority had a history of mental health difficulties including depression, psychosis and PTSD. Overall however, these figures are likely to reflect only the most severe cases in which the mental health difficulties were sufficiently prominent to be identified and recorded by detention staff. Moreover, they are likely to represent the perspective of detention staff rather than of asylum seekers themselves.

\section{Comparison studies}

Six studies compared the self-reported levels of PTSD, depression and anxiety of detainees to those in a comparison group of non-detained refugees or migrants from a similar background. All six studies showed higher symptom scores and rates of meeting clinical threshold for mental health disorders in the detained groups. This held true when comparing detained asylum seekers with non-detained asylum seekers [38, 41] detained asylum seekers and detained former prisoners with non-detained asylum seekers [11], detained sex workers with non-detained sex workers [37] and detained temporary protection visa holders with non-detained permanent protection visa holders [39, 43, 51]. All but one study [11] found no significant difference in levels of pre-migration trauma exposure between the groups, suggesting that detention contributed independently to PTSD over and above the impact of past traumatic experiences. However, whilst the number of traumatic events may not have differed across groups, the frequency, severity and context of those events were often not assessed.

Whilst no direct comparison between detainees and prison populations have been conducted to date, one study compared findings obtained from one immigration removal centre in the UK against results from prison-based studies utilising the same screening tools [45]. Despite methodological shortcomings and difficulties in making direct comparisons, prevalence rates of depression and PTSD were higher among immigration detainees than in prison populations. Levels of suicidal ideation were high in both groups.

\section{Detention duration and release}

Eight studies reported on the relationship between length of detention or release from detention and mental health and/or quality of life. Four studies conducted in Australia and the USA found a significant positive correlation between detention duration and symptomatology $[36,43,44,48]$, suggesting that psychological functioning deteriorates with prolonged detention. A UK study found an interaction effect between exposure to interpersonal trauma and length of detention, suggesting that experience of interpersonal trauma and prolonged time in detention resulted in higher anxiety and depression scores [11]. The authors suggest that the interaction they found may also reflect the complex relationship between stage of claim and detention duration within the UK. However, even brief detention (median 17.5 days) has been found to have negative mental health consequences [38].

In contrast, two studies did not find a statistically significant correlation between detention duration and quality of life [42, 46], although a decreasing trend was found in one of these studies [46]. Here, the small sample size as well as the use of quality of life scales rather than mental symptom measures may have resulted in reduced sensitivity to the effects of detention. Furthermore, one of the two 'negative' studies indicated a significant increase of suicidal ideation with detention duration [42].

One study employed a longitudinal design with a two-month follow up, indicating not only that detention exacerbates psychological symptoms over time, but also that these symptoms were markedly reduced in those participants who had been released prior to the follow-up rating [44]. However, another longitudinal study suggests that symptomatology and social isolation remained higher in former detainees following release when compared to their non-detained counterparts [39].

Three studies conducting single assessments following release from detention suggest that symptoms of depression, anxiety and PTSD endure beyond the detention period, and persist at 10 months [41], 3 years [43] or almost 4 years [40] after release. In the two latter studies, numerous anxiety-related symptoms, such as avoidance of related triggers, nightmares and flashbacks were linked directly to the detention experience. The severity of such long-term impacts, along with continuing sadness, hopelessness, and anger were again found to correlate with detention duration [43].

Despite this, an improvement in quality of life following release was found when asking former detainees to give retrospective ratings of their quality of life during detention and current ratings following release from detention [42]. After release, participants exhibited more satisfaction, improved mood and a decline in suicidal ideation.

\section{Gender}

To date, an overwhelming proportion of research focuses on males in detention. In those studies in which 
female detainees were also included, analysis by gender was usually not conducted. In one study, females were deliberately excluded from the sample due to differing experiences in comparison to men [41].

Only two studies specifically examined gender differences, with one finding no significant differences between genders [38] and the other reporting higher rates of trauma/PTSD in females [48]. Female detainees were also found to be more vulnerable to the effects of more prolonged detention when compared to males.

One study concentrated exclusively on female sex workers in detention, finding high rates of substance abuse, depression, somatic symptoms and PTSD amongst the women [37]. However, this sample of Russian sex workers in Israel differs substantially from other groups of detainees, complicating the generalizability and comparability of these findings. The mental health consequences of detention for female detainees specifically therefore remain unknown based on the studies reviewed.

\section{Children and families}

Ten studies reported data on the mental health of children and/or families within the context of detention. This review reports on 629 children and young people (across 9 studies) and 302 parents (across 5 studies).

Table 4 Prevalence Rates of Mental Health Difficulties in Children and Families

\begin{tabular}{|c|c|c|c|c|c|c|}
\hline & \multicolumn{2}{|l|}{$\begin{array}{l}\text { Steel et al. [23] } \\
\text { Diagnostic Interview }\end{array}$} & \multicolumn{2}{|c|}{$\begin{array}{l}\text { Mares \& Jureidini [53] } \\
\text { Diagnostic Interview }\end{array}$} & \multicolumn{2}{|c|}{$\begin{array}{l}\text { Lorek et al. [52] } \\
\text { Diagnostic Interviews, Parent-report, } \\
\text { Self-report }\end{array}$} \\
\hline & $\begin{array}{l}\text { Prevalence Rates } \\
\text { in Children ( } N= \\
\text { 20) }\end{array}$ & $\begin{array}{l}\text { Prevalence Rates } \\
\text { in Parents }(N=14)\end{array}$ & $\begin{array}{l}\text { Prevalence Rates } \\
\text { in Children ( } N= \\
\text { 20) }\end{array}$ & $\begin{array}{l}\text { Prevalence Rates } \\
\text { in Parents }(N=16)\end{array}$ & $\begin{array}{l}\text { Prevalence Rates } \\
\text { in Children }(\mathrm{N}= \\
\text { 11) }\end{array}$ & $\begin{array}{l}\text { Prevalence Rates } \\
\text { in Parents }(N=9)\end{array}$ \\
\hline \multicolumn{7}{|l|}{ Mental Health Difficulty } \\
\hline $\begin{array}{l}\text { Major Depressive } \\
\text { Disorder }\end{array}$ & $95 \%$ & $100 \%$ & $100 \%$ & $87 \%$ & $100 \%$ & $100 \%$ \\
\hline $\begin{array}{l}\text { Post-traumatic Stress } \\
\text { Disorder }\end{array}$ & $50 \%$ & $86 \%$ & $100 \%$ & $56 \%$ & $17 \%$ & $55 \%$ \\
\hline Anxiety Disorder & $\begin{array}{l}\text { 50\% (separation } \\
\text { anxiety) }\end{array}$ & $100 \%$ & $70 \%$ & - & $36-100 \%$ & $100 \%$ \\
\hline Sleep Related Difficulties & $65 \%$ & - & $100 \%$ & - & $91 \%$ & - \\
\hline $\begin{array}{l}\text { Eating Related } \\
\text { Difficulties }\end{array}$ & - & - & - & - & $100 \%$ & - \\
\hline $\begin{array}{l}\text { Oppositional Defiant } \\
\text { Disorder / Conduct } \\
\text { Problems }\end{array}$ & $45 \%$ & - & $30 \%$ & - & $55 \%$ & - \\
\hline Psychotic Symptoms & - & $14 \%$ & - & $25 \%$ & - & - \\
\hline Suicidal Ideation & $+50 \%$ & $93 \%$ & - & - & - & $100 \%$ \\
\hline Self-harm & $25 \%$ & $36 \%$ & $80 \%$ & $31 \%$ & - & - \\
\hline Somatic Complaints & - & - & $50 \%$ & - & $91 \%$ & - \\
\hline Hyperactive Behaviour & - & - & - & - & $27 \%$ & - \\
\hline $\begin{array}{l}\text { Peer Relationship } \\
\text { Problems }\end{array}$ & - & - & - & - & $64 \%$ & - \\
\hline \multicolumn{7}{|l|}{ Developmental Concerns } \\
\hline Enuresis & $\begin{array}{l}50 \% \text { of children in } \\
\text { middle childhood }\end{array}$ & n.a. & $\begin{array}{l}30 \% \text { of children } \\
\text { aged 6-17 }\end{array}$ & - & $\begin{array}{l}13 \% \text { of children } \\
\text { aged } 1-4\end{array}$ & n.a. \\
\hline Encopresis & - & n.a. & - & - & $\begin{array}{l}13 \% \text { of children } \\
\text { aged } 1-4\end{array}$ & n.a. \\
\hline Food Refusal & - & n.a. & - & - & $\begin{array}{l}38 \% \text { of children } \\
\text { aged } 1-4\end{array}$ & n.a. \\
\hline Return to Nappies & - & n.a & - & - & $\begin{array}{l}38 \% \text { of children } \\
\text { aged } 1-4\end{array}$ & n.a. \\
\hline Language Regression & - & n.a. & $\begin{array}{l}50 \% \text { of children } \\
\text { aged under } 5\end{array}$ & - & $\begin{array}{l}50 \% \text { of children } \\
\text { aged } 1-4\end{array}$ & n.a. \\
\hline Attachment Problems & - & n.a. & $\begin{array}{l}30 \% \text { of children } \\
\text { aged under } 5\end{array}$ & n.a. & - & n.a. \\
\hline
\end{tabular}


Two studies were conducted as comparison studies, with a total of 96 controls. Seven studies were conducted while participants were held in detention, and three were conducted following detention. Again, detention duration was differentially reported. Where reported, the means fell between 6 months and 2 years and 4 months and the ranges spanned from 11 days to 2 years and 8 months across studies. The ages of children spanned from childhood to adolescence, ranging from 11 months - 19 years. Both male and female children were represented, yet gender distributions were largely unreported.

Overall, 5 studies used either self-report or parent/ teacher report measures Two studies combined clinical assessment with self-report measures to evaluate psychiatric vulnerability $[23,52]$. This allowed not only for an estimation of prevalence rates of psychiatric disorders, but also for retrospective accounts of mental health difficulties preceding detention. One study reported clinical outcomes of referrals into Child and Adolescent Mental Health Services (CAMHS) from a detention centre [53]. Two further studies reported on data collected as part of an Australian Human Rights Commission Data Set [48, 54].

A wide range of psychological disturbances mirroring and extending those of adults was found across all three studies which also all included clinical assessments (see Table 4). All children evidenced at least one psychiatric disorder, most frequently depression, anxiety, PTSD and somatization, depending on the diagnostic categories used. The overwhelming majority also struggled with sleeping (65-100\%) and eating problems (100\%), suicidal ideation (50\%), and self-harm (25-80\%). Drawing on data from telephone-administered psychiatric interviews and self-report measures from a near-complete sample of detained families of unspecified ethnicity, Steel et al. [23] found psychiatric disorders to be ten times more likely to occur subsequent to detention when compared to prior to detention. Although based on retrospective reports, such difficulties appeared to be specifically linked to detention experiences. Combining clinical assessments with parent-report and, where possible, triangulating these with self-report measures of mental health in a small sample of child and adolescent detainees, Lorek et al. [52] further identified age-related differences in mental health difficulties. Specifically, whilst elevated levels of emotional and behavioural problems and somatic complaints were common across all age groups, developmental delay and regression were more apparent in younger detainees (aged 3-6). These included frequent crying and withdrawal (100\%), disturbed sleep routines $(100 \%)$, language delays $(50 \%$,$) , loss of previ-$ ously acquired cognitive skills (33\%) return to nappies (38\%), enuresis (13\%) and encopresis (13\%). Amongst older children (aged 7-11), self-report questionnaires suggested high levels of depression $(100 \%$ not within normal range, $50 \%$ above the cut off for clinical depression), anxiety (66\% above cut off for clinical anxiety) and PTSD symptomatology (17\% approaching clinical cut off) [52]. Though this study did not have a control group, the reported lack of pre-existing mental health problems again suggests that sudden mental health deterioration may be attributable to detention experiences.

Similar developmental differences in psychiatric morbidity were also found in the study of CAMHS assessments of 10 detained families [53]. Developmental concerns such as language regression (50\%), attachment problems (30\%) and enuresis (30\%) were reported in children under 5 years of age, whereas symptoms of PTSD (100\% above clinical cut off), depression with suicidal ideation (100\% above clinical cut-off), self-harm (80\%) anxiety (70\%), and somatic symptoms (50\%) were more common in older children (aged 6-17).

Regressive behaviours such as enuresis (45\% boys, $48 \%$ girls) and encopresis ( $8 \%$ boys, $11 \%$ girls) were also self-reported by a group of adolescents referred into an infirmary during their time in detention in one study, alongside the more common symptoms of severe to very severe PTSD (very severe 94\% boys, 96\% girls; severe 6\% boys, $4 \%$ girls; Rothe et al., 2002a). Following release from detention, $57 \%$ of adolescents within the same detention facility were considered to continue to meet a diagnosis of PTSD [56].

A secondary analysis of an Australian Human Rights Commission data set indicated very high rates of severe psychological distress amongst adolescents, evidenced by severe disorders for $85.7 \%$ of adolescents [54]. All met criteria for mixed anxiety and depression. Additionally, parent reports suggested a high probability of psychiatric disorder in $75 \%$ of their children, and significant emotional symptoms int $78.6 \%$. Drawing on a different subset of the collected data, it was estimated that a third of detained children had sufficient clinical symptoms to necessitate tertiary outpatient assessments [48].

\section{Comparison studies}

Two studies compared the mental health of children within the detention context against those outside it; both found elevated psychological symptoms within the detained groups [57, 58]. When comparing children of detained or deported parents with peers whose parents were either legal permanent residents or undocumented yet without prior contact with immigration enforcement, parent reports indicated higher levels of PTSD symptoms and trauma in children of detained or deported parents than the other two groups [57]. Parents and clinicians also rated detained children as scoring more highly on internalising problems and negative moods. However, the grouping of detention with deportation in 
this study makes it difficult to tease out the specific effects of the experience of detention as against those of removal or deportation. The authors concluded that forced separation from parents due to immigration enforcement may be particularly adverse to child mental health.

Similarly, a study comparing detained children to child asylum seekers in the community found detained children to have significantly impaired social-emotional wellbeing as measured by conduct disorder, emotional problems and hyperactivity than their non-detained counterparts [58]. Interestingly, detained children scored higher on peer relations and pro-social subscales, which may be essential skills for children living in challenging circumstances to have, particularly if their parents are unable to adequately meet their needs.

\section{Parental mental health}

Parents displayed high rates of psychopathology across the three studies (see Table 3). There was a substantial increase in reported suicidal ideation. Further, whilst in one study all parents reported the ability to care adequately for and support their children prior to detention, only one of 14 parents remained able to do so during detention [23].

One study reported on the effects of actual or threatened detention and deportation on family units using self-report measures [59]. Multiple regression analysis revealed that parents' legal vulnerability has an impact on the family environment including the quality of the parent-child relationship (27.1\% of variance), as well as on overall child well-being (30.6\% of variance). In other words, the greater the parents' legal vulnerability, the greater the reported consequences of detention and deportation. However, the interchangeable use of the terms 'detention' and 'deportation' again make it difficult to discern the specific effect of detention.

In keeping with reports on children and adolescents, the secondary analyses revealed $83 \%$ of parents to have severe disorders [54]. Again, all participants met criteria for mixed anxiety and depression. When asked about concerns surrounding their child's emotional wellbeing, mental health and development, $67 \%$ identified concerns around emotional and mental health (e.g. nightmares, worry, sadness) and 23\% identified concerns around development (e.g. poor eating and low weight).

\section{Detention duration and release}

Four studies investigated the relationship between detention duration and mental health difficulties. One study found that at 12-month follow up, the mental health of families who remained in detention further deteriorated during incarceration [53]. The three remaining studies did not find a significant correlation between length of detention and mental health problems [54, 56, 58]. However, the authors concluded that this might be due to small sample sizes, the lack of available data on detention duration as well as the possibility of a ceiling effect demonstrating extensive mental health difficulties.

\section{Discussion}

\section{Key findings of this review}

Adverse mental health consequences of immigration detention are consistently recognised across the literature. Such findings prevail even in countries where detention standards are regarded as relatively benign $[46,52]$. Much of the clinical literature reports high levels of anxiety, depression and PTSD and poor quality of life. Whilst such mental health difficulties cannot be viewed in isolation from past histories and pre-detention traumas, which are not consistently measured across studies, controlled studies with non-detained controls uniformly suggest greater symptoms and 'caseness' (i.e. meeting diagnostic criteria for a specific mental health condition) in detained samples. This indicates that detention plays an independent role in contributing to poor mental health outcomes amongst asylum seekers. Nonetheless, being an asylum seeker or having greater trauma exposure of any kind (whether torture or other exposure) prior to detention seems to be associated with higher rates of anxiety, depression and PTSD in the context of such detention (e.g. [41]). Similarly, greater isolation from families and living alone has been found to increase such symptoms in immigration detainees (e.g. [43]).

All adult studies examining the association between detention duration and mental health severity $(N=5)$ demonstrate a significant relationship between detention duration and mental health deterioration. No such correlation was found for length of detention and quality of life $(N=2)$, although a negative trend was found in one of these studies [46]. Additionally, perceived support from detention staff was found to impact positively on quality of life, highlighting the importance of staff behaviour on the wellbeing of detainees but not necessarily having any effect on their mental health problems. Symptom severity has been found to decrease and quality of life to increase following release, but mental health difficulties persist well beyond release. Despite the prominence of mental health disorders amongst detainees, significant barriers to the identification and treatment of those needs remain [47].

The comparatively smaller body of research on detained children and families $(N=10$ and a total of 629 children studied) echoes the findings in adults summarised above. It should be noted however that the detention of children is fairly uncommon across several countries including the UK, yet remains relatively widely practiced in some European countries [60] and, as 
recently widely publicised, in the USA. Across those settings and jurisdictions where it remains permitted, profound and far-reaching mental health difficulties are found amongst detained children and young people. Only one in four studies found the severity of mental health difficulties to increase with time the children spent in detention [53]. The other three studies found no correlation between detention duration and mental health. While this has not been researched, this may be explained by the possibility that even small durations of detention are traumatic and harmful to children. The nature of the mental health difficulties found differs across age groups; whereas younger children display developmental regression and primarily externalising behaviours, symptoms of depression, anxiety and PTSD are prominent in older children. Psychosomatic symptoms or somatization appear to be common across developmental stages. This may reflect the difference in experiencing and communicating psychological difficulties in children and young people [61, 62], but may also be understood as a reflection of the somatic component within the symptom pattern of complex PTSD [63]. Whilst detention itself may compromise child development and psychosocial health, as well as aggravating existing trauma, it may also lead to a breakdown of family units. The detention environment may therefore undermine adequate parenting abilities and leave parents feeling disempowered, as they struggle with their own mental health difficulties. Taken together, this may prevent parents from being able to provide support necessary for healthy child development, leaving children at risk of adverse mental health outcomes [64]. It remains unclear however, whether detaining children together with their parents or forcibly separating children from their families is more harmful to the children's mental health. Further, it is currently unknown how rates of parental mental health difficulties compare to those of single adults and those whose families are not detained. Finally, there is a dearth of quantitative research on the mental health of unaccompanied minors who are detained. With the current number of unaccompanied minors rising across the globe, research into the effects of immigration detention on this subgroup of children is of high importance, particularly with view to the possibility that some unaccompanied minors may be inappropriately treated as though they were adults, consequently increasing their risk of exploitation and harm [65].

Overall, the findings suggest that detention exacerbates the mental health burden of asylum seekers and refugees and that such detention should be viewed as a traumatic experience in and of itself. This may be particularly true for those detainees who are particularly vulnerable prior to detention. Individuals with pre-existing mental health difficulties may also be less effective self-advocates and therefore more likely to be detained [11], despite recommendations and policies to the contrary [31].

\section{Key strengths and limitations of this review}

A comprehensive search strategy, as well as independent screening, data extraction and quality appraisals were applied to ensure a systematic approach to synthesising the literature on the mental health consequences of immigration detention. Adherence to the PRISMA guidelines further increased the methodological rigour and clarity of the review. We sought to ensure that all research that met out criteria was identified through additional contact with expert researchers in the field. Finally, the exclusion of grey literature, as well as confining the search to papers written in English, inevitably resulted in the exclusion of some papers which may have limited the overall understanding of detainee mental health.

\section{What this review adds to existing reviews}

To the best of our knowledge, the current review is the first systematic review on the mental health of immigration detainees since our previous review published in 2009 [14]. Although excluding grey literature, it includes a larger number of clinical studies than previous reviews [30] and is not limited to detainees with a recorded history of torture [66]. The current review supports findings from the 2009 review and additionally highlights the importance of considering the impact of immigration detention on the mental health of families. It also includes multiple comparison studies which highlight the independent effect of such detention on mental health over and above that of uncertain immigration status. However, it also highlights the dearth (and consequent future importance) of further high quality research directly investigating vulnerability within the immigration detention context.

\section{Key limitations of the available evidence and their implications for future research}

Numerous methodological problems exist at primary study level, which may restrict the conclusions that can be drawn in this review but also indicate where further research is needed. The evidence to date remains sparse due to the multiple obstacles hindering such research. These include restricted access opportunities into detention facilities as well as the prohibiting of random sampling (e.g. [38, 44]). This may have led to an increased reliance on qualitative rather than quantitative methods, particularly in the study of detained children. Such studies have not been included in this review. With regards to studies conducted within detention centres, participants are often recruited through community areas within the detention centres (e.g. [11]), possibly introducing a 
bias towards those detainees who are most healthy. Recruitment of participants in studies conducted following release from detention, often occurs through lawyers, community organisations or other support services (e.g. [41]). Most studies did not describe the detention regime in sufficient detail to allow for this to be considered as a potential influencing factor. This, together with clearer differentiation between the traumatic effects of detention itself from those of the detention environment) is an important focus for future research. It also carries the implication that future studies should provide as much information as possible about the detention environment in order to facilitate judgment as to the generalisability of the findings. Participation rates are often unknown. It is therefore possible that the recruited samples differed systematically from detainees who did not access community facilities or come into contact with support services. Samples may therefore represent only the most vulnerable individuals in need of support, or conversely, the most highly functioning who are able to access appropriate services. Further research in more representative populations is needed to allow more confident generalisation of the findings to the detainee population as a whole. Additionally, the probability of being detained depends to some extent to sex and nationality, resulting in predominantly male populations of certain national origins [38]. Whilst a broad range of nationalities is represented across studies, a paucity of research focusing on women is evident. Differing nationalities contribute to the vast heterogeneity of samples, along with varying past experiences and traumatic histories, migration statuses, and detention systems and immigration policies within host countries. Correspondingly, language may be an important complicating factor, acting both as a prominent exclusion criterion or a possible cause of misinterpretation or inability to express mental health difficulties clearly [47]. Communicating mental health problems may additionally be compounded by differing expressions of distress and trauma, alongside differing attitudes and experiences of mental health within countries of origin [67]. However, participants from a wide range of cultural backgrounds seem to endure similar psychological ill-health when subjected to detention.

Multiple designs as well as a diversity of methodology and instruments used are evident across studies. Whilst these may differentially contribute to the overall understanding of mental health problems amongst detainees, they also limit comparability. The majority of research is conducted as cross-sectional studies, which warrant caution in the drawing of casual inferences. The suggested causal link between detention and worsening of mental health is however consistent with a limited number of longitudinal studies and comparison studies, implying that detention not only exacerbates existing mental health disorders, but also contributes independently to the onset of new ones. Nonetheless, isolating the effects of detention alone remains a complex task and additional longitudinal research is needed in order to establish the mental health trajectories of detainees, from the very early days of being arrested and detained. This may also include investigating the adverse effects of being detained multiple separate times at different lengths, as well as the effects that experiences in detention may have on people's ability to navigate and integrate into new societies. Finally, the frequent reliance on a range of self-report questionnaires, which have often not been validated specifically for the use with detainee populations, may be problematic though it is in our view unlikely that this significantly affects the overall validity of the findings. Moreover, measures often focus solely on symptoms of PTSD, depression and anxiety, which may fail to investigate other disorders such as schizophrenia and other non-affective disorders as may be the case in refugees as a whole [5]. Whilst self-report questionnaires are undoubtedly useful for the purpose of screening for mental health problems, outcomes of such self-report measures should not be equated with formal psychiatric diagnoses, which require clinician-administered interviews. To date, there is a limited use of diagnostic clinical assessments to complement self-report measures, and where these are conducted, the samples are restricted to adult male detainees only.

\section{Conclusions and practical recommendations}

Taken as a whole, the body of research reviewed in this paper consistently illustrates severe mental health consequences amongst detainees across a wide range of settings and jurisdictions. In view of this, in terms of provision of care, consideration of mental health disorders and their sequelae should become routine in making the decision whether or not to detain, and in monitoring the welfare of those already in detention. Comprehensive sociocultural assessments should be carried out by care providers with transcultural knowledge, skills and practices [68]. These assessments should lead to adequate treatment for individuals in detention who suffer from mental health difficulties. Additionally, there is a need to identify and address vulnerability within an overall asylum policy and more specifically a detention policy that avoids or minimises further harm being done to vulnerable people. For this, it is important to take into account multiple varying expressions of distress and vulnerability, which should not be reduced to Western conceptualisations. Moreover, a broad concept of past trauma is needed which is not restricted to the definition of torture according to Article 1 of the Convention Against Torture [69], but rather focuses on the severity of harm to which the individual has been subjected [70]. Further, there should be a greater focus on minimizing length of detention. Our findings add weight to the call 
in the UK and other countries to introduce a statutory limit of duration of detention, such as exists in all other EU countries [71]. The increasing evidence for the negative impact of immigration detention on vulnerable populations is particularly policy-relevant. Similarly, there is insufficient attention paid to child protection and safety, or to adequate education and health protection in countries where children continue to be detained, despite the recognised vulnerability and risk of children and young people and their protection under the UN Convention on the Rights of the Child [72].

\section{Additional file}

Additional file 1: Systematic Review Immigration Detention.

(DOCX $29 \mathrm{~kb}$ )

\section{Abbreviations}

CAMHS: Child and Adolescent Mental Health Service; EU: European Union; PTSD: Post-traumatic stress disorder; UK: United Kingdon; UNHCR: United Nations High Commissioner for Refugees; USA: United States of America; WHO: World Health Organization

\section{Acknowledgements}

We are grateful to Erin Dexter and Dafni Katsampa for their help in preparing this paper for final submission.

\section{Funding}

This study was supported by a research grant to the Helen Bamber Foundation from the Oak Foundation (Grant number: OCAY-15-286).

\section{Availability of data and materials}

Not applicable since no new data involved.

\section{Authors' contributions}

MvW, KR, ZC, RS, LO, CM and CK contributed to the conception and design of the review. ZC,RS and CM undertook the systematic search, screening and selecting articles. MvW, CM and CK assessed study quality. MvW interpreted the data and drafted and revised all versions of the manuscript. KR, ZC, RS, CM and CK critically reviewed the manuscript. LO independently reviewed the drafts. All authors have read and approved the final manuscript.

\section{Authors' information}

The Helen Bamber Foundation (HBF), based in London, supports people who have experienced extreme human cruelty, such as torture or human trafficking, who are refugees and asylum seekers. We are a human rights charity with clinical and legal expertise and we deliver a specialist Model of Integrated Care (MolC) to meet the complex needs of some of the most vulnerable people in the world. HBF's experts deliver this MolC tailored to each client which includes:

- Clinical: specialist psychological care; medical advisory services and physical rehabilitation therapies

- Legal protection and medico-legal assessment and documentation

- Safeguarding and prevention of social deprivation

- Community integration through life, employment and creative skills

We support the most marginalised people in our society to secure their needs and rebuild their lives. We amplify the impact of our work to benefit survivors more widely through advocating for policy change, research, and dissemination of best practice.

Ethics approval and consent to participate Not applicable.
Consent for publication

Not applicable.

\section{Competing interests}

The authors declare that they have no competing interests.

\section{Publisher's Note}

Springer Nature remains neutral with regard to jurisdictional claims in published maps and institutional affiliations.

\section{Author details}

${ }^{1}$ Helen Bamber Foundation, Bruges Place, 15-20 Baynes Street, London NW1 OTF, UK. ${ }^{2}$ Health Services and Population Research Department, Kings College London, London, UK. ${ }^{3}$ Division of Psychiatry, University College London, London, UK.

Received: 22 September 2017 Accepted: 25 October 2018

Published online: 06 December 2018

References

1. The United Nations High Commissioner For Refugees. Figures at a glance. 2017. http://www.unhcr.org/uk/figures-at-a-glance.html. Accessed 17 Sept 2017.

2. Global Detention Project. https://www.globaldetentionproject.org/ organisations-alliances/european-union-eu. Accessed 17 Sept 2017.

3. Fazel M, Wheeler J, Danesh J. Prevalence of serious mental disorder in 7000 refugees resettled in western countries: a systematic review. Lancet. 2005; 365:1309-14.

4. Priebe S, Giacco D, El-Nagib R. Health evidence network synthesis report 47. Public health aspects of mental health among migrants and refugees: A review of the evidence on mental health care for refugees, asylum seekers and irregular migrants in the WHO European Region. Geneva: World Health Organization; 2016.

5. Hollander AC, Dal H, Lewis G, Magnusson C, Kirkbride JB, Dalman C. Refugee migration and risk of schizophrenia and other non-affective psychoses: Cohort study of 1.3 million people in Sweden. BMJ. 2016;352:11030.

6. Song SJ, Kaplan C, Tol WA, Subica A, de Jong J. Psychological distress in torture survivors: pre-and post-migration risk factors in a US sample. Soc Psych Psych Epid. 2015;50:549-60.

7. Schick M, Zumwald A, Knöpfli B, Nickerson A, Byrant RA, Schnyder U, et al. Challenging future, challenging past: the relationship of social integration and psychological impairment in traumatized refugees. Eur J Psychotraumatol. 2016;7:28057.

8. Laban CJ, Gernaat HB, Komproe IH, van der Tweel I, de Jong JT. Postmigration living problems and common psychiatric disorders in Iraqi asylum seekers in the Netherlands. J Nerv Ment Dis. 2005;193:825-32.

9. Warfa N, Curtis S, Watters C, Carswell K, Ingleby D, Bhui K. Migration experiences, employment status and psychological distress among Somali immigrants: A mixed-method international study. BMC Public Health. 2012;12:749

10. Carswell K, Blackburn P, Barker C. The relationship between trauma, postmigration problems and the psychological well-being of refugees and asylum seekers. Int J Soc Psychiatr. 2011;57:107-19.

11. Robjant K, Robbins I, Senior V. Psychological distress amongst immigration detainees: a cross-sectional questionnaire study. Br J Clin Psychol. 2009;48:275-86.

12. Medical Justice. http://www.medicaljustice.org.uk/about/what-we-do/ research-and-campaigning/publications/. Accessed 17 Sept 2017.

13. Schauer M, Neuner F, Karunakara U, Klaschik C, Robert C, Elbert T. PTSD and the 'building block' effect of psychological trauma among West Nile Africans. Eur J Psychotraumatol. 2003;10:5-6.

14. Robjant K, Hassan R, Katona C. Mental health implications of detaining asylum seekers: a systematic review. Brit J Psychiat. 2009;194:306-12.

15. Australian Association of Social Workers. Position Statement: Children in immigration detention. 2015. https://www.aasw.asn.au/document/item/ 7264. Accessed 17 September 2017.

16. Canadian Council for Refugees. Ending immigration detention of children: Call for legislative amendment. 2016. http://ccrweb.ca/sites/ccrweb.ca/files/ ending-detention-children-proposal.pdf. Accessed 17 Sept 2017.

17. American Orthopsychiatric Association. The impact of immigration enforcement on children and families. 2010. http://www.bhjustice.org/ assets/docs/positionstatements/immigration\%20enforcement.pdf. Accessed 17 Sept 2017. 
18. The United Nations High Commissioner For Refugees. UNHCR deeply concerned by Hungary plans to detain all asylum seekers. 2017. http://www.unhcr.org/uk/news/briefing/2017/3/58be80454/unhcrdeeply-concerned-hungary-plans-detain-asylum-seekers.html. Accessed 17 September 2017.

19. European Commission. The use of detention and alternatives to detention in the context of immigration policies. Synthesis report for the EMN Focused Study 2014 https:/ec.europa.eu/home-affairs/sites/homeaffairs/files/what-we-do/networks/ european_migration_network/reports/docs/emn-studies/emn_study_detention_ alternatives_to_detention_synthesis_report_en.pdf. Accessed 17 Sept 2017.

20. Immigration Detention. Human Rights First. 2018. https://www. humanrightsfirst.org/topics/immigration-detention. Assessed 22 Jul 2018

21. USA Today. 2018. https:/eu.usatoday.com/story/news/2018/06/27/immigrantchildren-family-separation-border-timeline/734014002/. Accessed 27 June 2018.

22. Sultan A, O'Sullivan K. Psychological disturbances in asylum seekers held in long term detention: a participant-observer account. Med J Australia. 2001;175:593-6.

23. Steel Z, Momartin S, Bateman C, Hafshejani A, Silove DM, Everson N, et al. Psychiatric status of asylum seeker families held for a protracted period in a remote detention Centre in Australia. Aust NZ J Pub Heal. 2004;28:527-36.

24. Migration Amendment (Detention Arrangements) Act 2005. 2008 Legislation.gov.au https://www.legislation.gov.au/Details/C2005A00079. Accessed 22 Jul 2018.

25. Fazel M, Silove D. Detention of refugees. BMJ. 2006;332(7536):251-2.

26. Immigration Detention News, Research and Analysis - The Conversation. The Conversation. 2018. https://theconversation.com/topics/immigrationdetention-12212. Accessed 22 Jul 2018.

27. All Party Parliamentary Group on Refugees \& the All Party Parliamentary Group on Migration. The Report of the Inquiry into the Use of Immigration Detention in the United Kingdom. 2015. https://detentioninquiry.files.wordpress.com/2015/03/ immigration-detention-inquiry-report.pdf. Accessed 23 Jul 2018

28. The Royal College of Psychiatrists. Position Statement on detention of people with mental disorders in immigration removal centres. 2013. http://www. medicaljustice.org.uk/wp-content/uploads/2016/06/Appendix-A-The-RoyalCollege-of-Psychiatrists-Position-Statement-on-detention-of-people-with-mentaldisorders-in-Immigration-Removal-Centres.pdf. Accessed 28 Nov 2018

29. Shaw S. Review into the welfare in detention of vulnerable persons. 2016. https://www.gov.uk/government/uploads/system/uploads/attachment_data/ file/490782/52532_Shaw_Review_Accessible.pdf. Accessed 17 Sept 2017.

30. Bosworth M. Mental health in immigration detention: a literature review. Review into the welfare in detention of vulnerable persons, $\mathrm{cm} 9186$. London: HSMO; 2016.

31. Immigration Act 2016: Guidance on adults at risk in immigration detention. Home Office 2016. https://www.gov.uk/government/uploads/system/ uploads/attachment_data/file/547519/Adults_at_Risk_August_2016.pdf. Accessed 17 Sept 2017.

32. Royal College of Psychiatrists. Definition of torture in the context of immigration detention policy. Position Statement 07. 2016. http://www. rcpsych.ac.uk/pdf/PS07_2016.pdf. Accessed 17 Sept 2017.

33. The Detention Centre (Amendment) Rules 2018. 2018. Legislation.gov.uk. http// www.legislation.gov.uk/uksi/2018/411/article/2/made. Accessed 22 Jul 2018.

34. Moher D, Liberati A, Tetzlaff J, Altman DG. Preferred reporting items for systematic reviews and mental-analyses: the PRISMA statement. PLoS Med. 2009;6:E1000097.

35. Critical Appraisal Skills Programme. http://www.casp-uk.net/casp-toolschecklists. Accessed 17 Sept 2017.

36. Green JP, Eagar K. The health of people in Australian immigration detention centres. Med J Australia. 2010;192:65-70.

37. Cwikel J, Chudakov B, Paikin M, Agmon K, Belmaker RH. Trafficked female sex workers awaiting deportation: comparison with brothel workers. Arch Womens Ment Health. 2004;7:243-9.

38. Cleveland J, Rousseau C. Psychiatric symptoms associated with brief detention of adult asylum seekers in Canada. Can J Psychiatr. 2013;58: 409-16.

39. Steel Z, Momartin S, Silove D, Coello M, Aroche J, Tay KW. Two year psychosocial and mental health outcomes for refugees subjected to restrictive or supportive immigration policies. Soc Sci Med. 2011;72: 1149-56.

40. Coffey GJ, Kaplan I, Sampson RC, Tucchi MM. The meaning and mental health consequences of long-term immigration detention for people seeking asylum. Soc Sci Med. 2010;70:2070-9.
41. Ichikawa M, Nakahara S, Wakai S. Effect of post-migration detention on mental health among afghan asylum seekers in Japan. Aust NZ J Psychiat. 2006;40:341-6.

42. Sobhanian F, Boyle GJ, Bahr M, Fallo T. Psychological status of former refugee detainees from the Woomera detention Centre now living in the Australian community. Psychiatr Psychol Law. 2006:13:151-9.

43. Steel Z, Silove D, Brooks R, Momartin S, Alzuhairi B, Susljik I. Impact of immigration detention and temporary protection on the mental health of refugees. Brit J Psychiat. 2006;188:58-64.

44. Keller AS, Rosenfeld B, Trinh-Shevrin C, Meserve C, Sachs E, Leviss JA, et al. Mental health of detained asylum seekers. Lancet. 2003;362:1721-3.

45. Sen P, Arungnanaseelan J, Connell E, Katona C, Khan A, Morna P, et al. Mental health morbidity amongst people subjected to immigration detention in the UK. Epidemiol Psychiatr Sci. 2017. https://doi.org/10.1017/ S2045796017000269.

46. Puthoopparambil SJ, Bjerneld M, Kaellestal C. Quality of life among immigrants in Swedish immigration detention centres: a cross-sectional questionnaire study. Glob Health Action. 2015;8:28321.

47. Graf M, Wermuth $P$, Haefeli D, Weisert A, Reagu S, Pflueger M, et al Prevalence of mental disorders among detained asylum seekers in deportation arrest in Switzerland and validation of the brief jail mental health screen BJMHS. Int J Law Psychiat. 2013;36:201-6.

48. Young $P$, Gordon M. Mental health screening in immigration detention: a fresh look at Australian government data. Aust Psychiatry. 2016;24(1):19-22.

49. Hedrick K. Getting out of (self-) harm's way: a study of factors associated with self-harm among asylum seekers in Australian immigration detention. J Forensic Legal Med. 2017;49:89-93.

50. Cohen J. Safe in our hands?: a study of suicide and self-harm in asylum seekers. J Forensic Legal Med. 2008;15(4):235-44.

51. Momartin S, Steel Z, Coello M, Aroche J, Silove DM, Brooks R. A comparison of the mental health of refugees with temporary versus permanent protection visas. MJA. 2006;185(7):357-61.

52. Lorek A, Ehntholt K, Nesbitt A, Wey E, Githinji C, Rossor E, et al. The mental and physical health difficulties of children held within a British immigration detention Centre: a pilot study. Child Abuse Negl. 2009;33:573-85.

53. Mares S, Jureidini J. Psychiatric assessment of children and families in immigration detention - Clinical, administrative and ethical issues. Aust NZ Pub Heal. 2004;28:520-6.

54. Mares S. The mental health of children and parents detained on Christmas Island: secondary analysis of an Australian human rights commission data set. Health Hum Rights. 2016;18(2):219-32.

55. Rothe EM, Castillo-Matos H, Busquets R. Post-traumatic stress symptoms in Cuban adolescent refugees during camp confinement. Adolescent Psychiatry. 2002a;26:97.

56. Rothe E, Lewis J, Castillo-Matos H, Martinez O, Busquets R, Martinez I. Posttraumatic stress disorder among Cuban children and adolescents after release from a refugee camp. Psychiatr Serv. 2002b;53(8):970-6.

57. Rojas-Flores L, Clements M, Hwang Koo J, London J. Trauma and psychological distress in Latino citizen children following parental detention and deportation. Psychol Trauma Theory Res Pract Policy. 2017;9(3):352-61.

58. Zwi K, Mares S, Nathanson D, Tay A, Silove D. The impact of detention on the social-emotional wellbeing of children seeking asylum: a comparison with community-based children. Eur Child Adolesc Psychiatry. 2017;27(4): 411-22.

59. Brabeck KM, Xu Q. The impact of detention and deportation on Latino immigrant children and families: a quantitative exploration. Hispanic J Behav Sci. 2010;32:341-61.

60. End Immigration detention of children. https://www.hrw.org/world-report/ 2016/children-behind-bars. Accessed 17 Sept 2017

61. Campo VJ. Annual research review: functional somatic symptoms and associated anxiety and depression - developmental psychopathology in pediatric practice. J Child Psychol Psyc. 2012:53:575-93.

62. Van der Kolk BA. The body keep the score: memory and the evolving psychobiology of posttraumatic stress. Harvard Rev Psychiat. 1994;1:253-65.

63. Cloitre M, Courtois CA, Ford JD, Green BL, Alexander P, Briere J, et al. The ISTSS Expert Consensus Treatment Guidelines for Complex PTSD in Adults. 2012. http://www.istss.org/ISTSS_Main/media/Documents/ISTSS-ExpertConcesnsus-Guidelines-for-Complex-PTSD-Updated-060315.pdf. Accessed 17 Sept 2017. 
64. International Detention Coalition. Captured Childhood. 2012. https:// idcoalition.org/wp-content/uploads/2012/03/Captured-Childhood-FINALJune-2012.pdf. Accessed 17 Sept 2017.

65. Zwi K, Mares $\mathrm{S}$. Stories from unaccompanied children in immigration detention: a composite account. J Paediatr Child H. 2015;51:658-62.

66. Storm T, Engberg $\mathrm{M}$. The impact of immigration detention on the mental health of torture survivors is poorly documents - a systematic review. Dan Med J. 2013;60:A4728.

67. Summerfield D. The invention of post-traumatic stress disorder and the social usefulness of a psychiatric category. BMJ. 2001;322:95-8.

68. Wylie L, Van Meyel R, Harder H, Sukhera J, Luc C, Ganjavi H, Elfakhani M, Wardrop N. Assessing trauma in a transcultural context: challenges in mental health care with immigrants and refugees. Public Health Rev. 2018;39(1):22.

69. United Nations Human Rights Office of the High Commisioner. Convention against torture and other cruel, inhumane or degrading treatment or punishment. 1987. http://www.ohchr.org/EN/Professionallnterest/Pages/CAT. aspx. Accessed 17 Sept 2017.

70. The Royal College of Psychiatrists. Position Statement 07. Definition of torture in the context of immigration detention policy. 2016. http://www. rcpsych.ac.uk/pdf/PS07_2016.pdf. Accessed 17 Sept 2017.

71. All Party Parliamentary Group. Refugees welcome? The experience of new refugees in the UK. 2017. https://www.refugeecouncil.org.uk/assets/0004/ 0316/APPG_on_Refugees_-_Refugees_Welcome_report.pdf. Accessed 17 Sept 2017.

72. United Nations Human Rights Office of the High Commissioner. Convention on the Rights of the Child. 1989. http://www.ohchr.org/en/professionalinterest/ pages/crc.aspx. Accessed 17 Sept 2017.

Ready to submit your research? Choose BMC and benefit from:

- fast, convenient online submission

- thorough peer review by experienced researchers in your field

- rapid publication on acceptance

- support for research data, including large and complex data types

- gold Open Access which fosters wider collaboration and increased citations

- maximum visibility for your research: over $100 \mathrm{M}$ website views per year

At $\mathrm{BMC}$, research is always in progress.

Learn more biomedcentral.com/submissions 